\title{
Noncoherent Receiver for Decode-and-Forward Cooperative Systems with Multi-Antenna Equipped Destination
}

\author{
Ha X. Nguyen ${ }^{1}$, Cuu V. Ho ${ }^{2}$, Chan Dai Truyen Thai ${ }^{3}$ \\ ${ }^{1}$ School of Engineering, Tan Tao University, Tan Duc E-city, Duc Hoa, Long An, Vietnam \\ ${ }^{2}$ Department of Electronics and Telecommunications, Saigon University, Ho Chi Minh City, Vietnam \\ 3 Singapore University of Technology and Design (SUTD), Singapore
}

Correspondence: Ha X. Nguyen, ha.nguyen@ttu.edu.vn

Manuscript communication: received 26 June 2014, accepted 29 September 2014

\begin{abstract}
This paper studies a maximum energy selection receiver for an adaptive decode-and-forward (DF) cooperative wireless system with multiple-antenna equipped destination. In particular, the destination selects the maximum output from all the outputs of the square-law detectors to perform the detection of the transmitted information. A close-form expression for the bit-error-rate (BER) is analytically derived when the system is deployed with binary frequency-shift keying (BFSK) modulation. The thresholds used at the relays to address the issue of error propagation are optimized to minimize the BER. While finding the optimal thresholds requires information on the average signal-to-noise ratios (SNRs) of all the transmission links in the system, the approximate thresholds at each relay that require only information on the average SNR of the source-corresponding relay are investigated. It is also derived that the system achieves a full diversity order with the approximate thresholds. Finally, both analytical and simulation results are provided to verify our analysis.
\end{abstract}

Keywords- cooperative diversity, frequency-shift keying, fading channel, decode-and-forward protocol, selection combining, square-law detector.

\section{INTRODUCTION}

Frequency shift keying (FSK) is a popular modulation scheme in noncoherent communications in which the receiver does not require any channel state information (CSI) to decode the transmitted signals [1]. Consequently, using FSK signals in cooperative systems has been focused recently since there is a complexity advantage in decoding [2-7]. It is due to the fact that there are many wireless fading channels involved in the systems [8,9], which makes the task of channel estimation more difficult. With the decode-and-forward (DF) protocol employing FSK in cooperative systems, reference [3] proposed maximum likelihood (ML) and suboptimal piecewise linear (PL) schemes to decode the signals at the destination. However, it was shown that the system could not achieve a full diversity order due to the error forwarding at the relays. References $[6,7]$ proposed to use a threshold at the relays to address the issue of error propagation for binary frequencyshift keying (BFSK) modulation. While the destination in [6] combines all the signals from the retransmitting relays, the destination in [7] selects only one signal with the largest magnitude of the energy difference to decode. Unfortunately, designing the optimal thresholds to minimize the average bit-error-rate (BER) of the system relies on the MATLAB Optimization Toolbox and a theoretical analysis of the diversity order is not available. Reference [10] proposes to employ the maximum energy selection (MES) receiver, i.e., selecting the maximum output from the square-law detectors of all branches to perform a detection, for a thresholdbased (i.e., adaptive) DF cooperative system. The results in [10] show that the system achieves a full diversity order with the approximate thresholds.

This work is a further development of [10]. It is concerned with a threshold-based DF cooperative system in which the destination is equipped with multiple antennas. In particular, after receiving the signal from the source in the first phase, each relay decides to retransmit the decoded information if its decision variable is higher than a threshold. Otherwise, it remains silent in the second phase. At the destination, maximum energy selection is employed to select the maximum output from the square-law detectors of all branches (i.e., source, relays, different antennas) to perform a detection. The average end-to-end bit-errorrate (BER) is analytically determined in a closed-form expression. Based on the BER expression, the use of the optimal thresholds at the relays is discussed to minimize the BER. The approximate thresholds that achieve full diversity are also provided. Note that the main difference between this paper and reference [10] is that the destination is equipped with multiple antennas. This leads to a study of a more generalized multiantenna DF relay network.

The remainder of this paper is organized as follows. Section 2 describes the system model. Section 3 presents the BER computation and discusses how to find the optimal and approximate thresholds. Numerical and simulation results are presented in Section 4. Finally, Section 5 concludes the paper. 


\section{System Model}

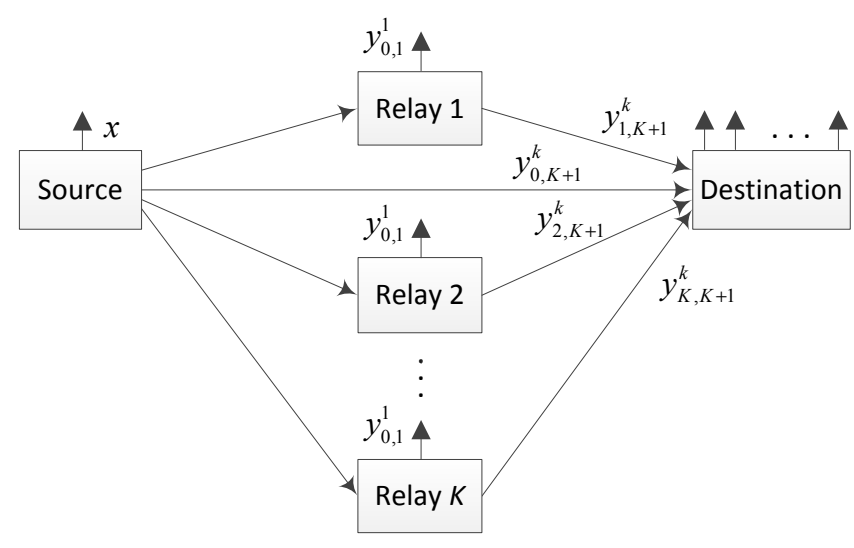

Figure 1. A wireless relay network.

We consider a cooperative relaying system in which the source, denoted by node 0 , communicates with the destination, denoted by node $K+1$, with the assistance of $K$ half-duplex relays, denoted by node $i, i=1, \ldots, K$, as illustrated in Figure 1. The source and the relays are equipped with a single antenna. Meanwhile, the destination is equipped with $N_{D}$ antennas. It is assumed that the $K$ relays retransmit signals to the destination over orthogonal channels. In this paper, we also assume that the fading channel coefficient between transmit node $i$ and the $k$ th receive antenna of node $j$, denoted by $h_{i, j}^{k}$ and the noise component at the $k$ th receive antenna of node $j$, denoted by $n_{i, j}^{k}$, are modeled as zero-mean complex Gaussian random variables with variances $\left(\sigma_{i, j}^{k}\right)^{2}$ and $N_{0}$, respectively. It is worth mentioning that the transmit node is always equipped with a single antenna. The instantaneous signal-to-noise ratio (SNR) of the channel between node $i$ and the $k$ th receive antenna of node $j$, which is denoted by $\gamma_{i, j}^{k}$, is given as $\gamma_{i, j}^{k}=E_{i}\left|h_{i, j}^{k}\right|^{2} / N_{0}$ where $E_{i}$ is the average transmitted energy of node $i$. The corresponding average SNR is $\bar{\gamma}_{i, j}^{k}=E_{i}\left(\sigma_{i, j}^{k}\right)^{2} / N_{0}$.

The signal transmission from the source to destination is illustrated in Figure 1. In the first phase, the source broadcasts the signal $x_{m}$ and the received signals at the $k$ th receive antenna of node $i, i=1, \ldots, K+1$, are written as

$$
y_{0, i}^{k}=\sqrt{E_{0}} h_{0, i}^{k} x_{m}+n_{0, i}^{k}, \quad i=1,2, \ldots, K+1
$$

where $x_{m}$ is the $m$ th symbol of an BFSK constellation. Note that since the relays are equipped with a single antenna and the destination is equipped with $N_{D}$ antennas, $k=1$ if $i=1,2, \ldots, K$ and $k=1,2, \ldots, N_{D}$ if $i=K+1$.

Similar to [10], signal detection at the $i$ th relay node is carried out by a square-law detector. Without loss of generality, assume that the the first symbol from the signal constellation is transmitted. The outputs of the square-law detector for the first and second symbols at node $i, i=1, \ldots, K$ are written, respectively, as

$$
\begin{aligned}
& y_{0, i, 1}^{1}=\left|\sqrt{E_{0}} h_{0, i}^{1}+n_{0, i, 1}^{1}\right|^{2}, \\
& y_{0, i, 2}^{1}=\left|n_{0, i, 2}^{1}\right|^{2} .
\end{aligned}
$$

As in $[6,10]$, the difference of the outputs of the square-law detector, namely $\theta_{0, i}=\left|y_{0, i, 1}^{1}-y_{0, i, 2}^{1}\right|$, is considered as a reliability measure of the detection at node $i$. Therefore, node $i$ decodes and retransmits a BFSK signal only if $\theta_{0, i}>\theta_{\mathrm{r}}^{\text {th }}$. When node $i$ transmits a correct bit in the second phase, the outputs of the square-law detector for the first and second symbols at the $k$ receive antenna of the destination are

$$
\begin{aligned}
y_{i, K+1,1}^{k} & =\left|\sqrt{E_{i}} h_{i, K+1}^{k}+n_{i, K+1,1}^{k}\right|^{2}, \\
y_{i, K+1,2}^{k} & =\left|n_{i, K+1,2}^{k}\right|^{2} .
\end{aligned}
$$

Meanwhile, the outputs of the square-law detector for the first and second symbols at the $k$ receive antenna of the destination can be written as follows if node $i$ transmits an incorrect bit:

$$
\begin{aligned}
y_{i, K+1,1}^{k} & =\left|n_{i, K+1,1}^{k}\right|^{2}, \\
y_{i, K+1,2}^{k} & =\left|\sqrt{E_{i}} h_{i, K+1}^{k}+n_{i, K+1,2}^{k}\right|^{2} .
\end{aligned}
$$

If $\theta_{0, i}<\theta_{\mathrm{r}}^{\text {th }}$, node $i$ remains silent in the second phase and the outputs of the square-law detector for the first and second symbols at the $k$ receive antenna of the destination are

$$
\begin{aligned}
& y_{i, K+1,1}^{k}=\left|n_{i, K+1,1}^{k}\right|^{2}, \\
& y_{i, K+1,2}^{k}=\left|n_{i, K+1,2}^{k}\right|^{2} .
\end{aligned}
$$

Finally, the destination compares and chooses the maximum output from all the outputs of the square-law detectors, i.e., employs the maximum energy selection (MES), to detect the transmitted information. In other words, the decision rule is of the following form:

$$
[\hat{i}, \hat{k}, \hat{m}]=\arg \max _{\substack{i=0, \ldots, K \\ k=1, \ldots, N N_{D} \\ m=1,2}} y_{i, K+1, m}^{k} .
$$

\section{BER COMPUtATIONS AND Thresholds}

In this section, the BER analysis for MES scheme is first carried out for a network with arbitrary qualities of source-relay and relay-destination links. Then, the optimal thresholds chosen to minimize the average BER are discussed. Finally, the approximate thresholds are proposed to achieve a full diversity order.

\subsection{BER Computations}

The law of total probability is employed to compute the average BER of the system. First, denote $\Omega_{1}, \Omega_{2}$, and $\Omega_{3}$ as the sets of the relays that forward a correct bit, an incorrect bit, and remain silent, respectively. It is clear that $K=\left|\Omega_{1}\right|+\left|\Omega_{2}\right|+\left|\Omega_{3}\right|$ where $\Omega \mid$ denotes the cardinality of set $\Omega$. The probability of occurrence for the specific set $\left\{\Omega_{1}, \Omega_{2}, \Omega_{3}\right\}$ is [7]:

$$
\begin{aligned}
P\left(\Omega_{1}, \Omega_{2}, \Omega_{3}\right)= & \prod_{i \in\left(\Omega_{1} \cup \Omega_{2}\right)}\left[1-I_{1}\left(\theta_{\mathrm{r}}^{\text {th }}, \bar{\gamma}_{0, i}^{1}\right)\right] \prod_{i \in \Omega_{3}} I_{1}\left(\theta_{\mathrm{r}}^{\text {th }}, \bar{\gamma}_{0, i}^{1}\right) \\
& \times \prod_{i \in \Omega_{1}}\left[1-I_{2}\left(\theta_{\mathrm{r}}^{\text {th }}, \bar{\gamma}_{0, i}^{1}\right)\right] \prod_{i \in \Omega_{2}} I_{2}\left(\theta_{\mathrm{r}}^{\text {th }}, \bar{\gamma}_{0, i}^{1}\right),
\end{aligned}
$$


where $A \cup B$ denotes the union of sets $A$ and $B$. The function $I_{1}\left(\theta_{\mathrm{r}}^{\text {th }}, \bar{\gamma}_{0, i}^{1}\right)$ is the probability of the event $\theta_{0, i}<$ $\theta_{\mathrm{r}}^{\text {th }}$ and is computed as [7]:

$$
\begin{aligned}
I_{1}\left(\theta_{\mathrm{r}}^{\mathrm{th}}, \bar{\gamma}_{0, i}^{1}\right)=\frac{1+\bar{\gamma}_{0, i}^{1}}{2+\bar{\gamma}_{0, i}^{1}}[1 & -\mathrm{e}^{\left.-\theta_{\mathrm{r}}^{\mathrm{th}} /\left(1+\bar{\gamma}_{0, i}^{1}\right)\right]} \\
& +\frac{1}{2+\bar{\gamma}_{0, i}^{1}}\left[1-\mathrm{e}^{-\theta_{\mathrm{r}}^{\mathrm{th}}}\right] .
\end{aligned}
$$

On the other hand, the function $I_{2}\left(\theta_{\mathrm{r}}^{\text {th }}, \bar{\gamma}_{0, i}^{1}\right)$ is the probability of error at node $i, i=1, \ldots, K$, given the event $\theta_{0, i}>\theta_{\mathrm{r}}^{\text {th }}$ and is determined by [7]

$$
I_{2}\left(\theta_{\mathrm{r}}^{\text {th }}, \bar{\gamma}_{0, i}^{1}\right)=\frac{1}{2+\bar{\gamma}_{0, i}^{1}} \frac{1}{1-I_{1}\left(\theta_{\mathrm{r}}^{\text {th }}, \bar{\gamma}_{0, i}^{1}\right)} \mathrm{e}^{-\theta_{\mathrm{r}}^{\text {th }}} .
$$

Now let $W_{w, m}^{k}\left(w \in\left\{\Omega_{1} \cup\{0\}\right\}\right), V_{v, m}^{k}\left(v \in \Omega_{2}\right)$ and $R_{r, m}^{k}\left(r \in \Omega_{3}\right)$ denote the outputs of the square-law detector for the $m$ th symbol, $m=1,2$, measured at the $k$ receive antenna of the destination. With the assumption that the the first symbol from the signal constellation is transmitted, the probability density functions (pdfs) of $W_{w, m}^{k}, V_{v, m}^{k}$ and $R_{r, m}^{k}$ are given, respectively, by

$$
\begin{aligned}
f_{W_{w, m}^{k}}(x) & = \begin{cases}f_{w, 1}^{k}(x), & m=1 \\
f_{w, 2}^{k}(x), & m=2\end{cases} \\
f_{V_{v, m}}^{k}(x) & = \begin{cases}f_{v, 2}^{k}(x), & m=1 \\
f_{v, 1}^{k}(x), & m=2\end{cases} \\
f_{R_{r, m}}^{k}(x) & =f_{r, 2}^{k}(x), \quad m=1 \text { or } m=2,
\end{aligned}
$$

where

$$
\begin{aligned}
& f_{h, 1}^{k}(x)=\frac{1}{N_{0}\left(1+\bar{\gamma}_{h, K+1}^{k}\right)} \mathrm{e}^{-x /\left(N_{0}\left(1+\bar{\gamma}_{h, K+1}^{k}\right)\right)}, x \geq 0 \\
& \text { and } \\
& f_{h, 2}^{k}(x)=\frac{1}{N_{0}} \mathrm{e}^{-x / N_{0}}, x \geq 0 .
\end{aligned}
$$

An error occurs at the destination if among the $2(K+$ 1) $N_{D}$ statistics $W_{w, m}^{k}, V_{v, m}^{k}$ and $R_{r, m}^{k}, w \in\left\{\Omega_{1} \cup\{0\}\right\}$, $v \in \Omega_{2}, r \in \Omega_{3}, k=1, \ldots, N_{D}, m=1,2$, the one with the largest value is 1$)$ Case $1(\Theta=1)$ one of $\left.W_{w, 1}^{k}, 2\right)$ Case $2(\Theta=2)$ one of $V_{v, 1}^{k}$, and 3) Case $3(\Theta=3)$ one of $R_{r, 1}^{k}$. Thus, given the set $\left\{\Omega_{1}, \Omega_{2}, \Omega_{3}\right\}$, the BER can be computed as

$$
\begin{aligned}
& P_{\Omega_{1}, \Omega_{2}, \Omega_{3}}(\varepsilon)=\sum_{i=1}^{3} P_{\Omega_{1}, \Omega_{2}, \Omega_{3}}(\varepsilon, \Theta=i) \sum_{k=1}^{N_{D}} \sum_{w \in \Omega_{1} \cup\{0\}} P\left(\bar{W}_{w, 2}^{k}-W_{w, 2}^{k}<0\right) \\
&+\sum_{k=1}^{N_{D}} \sum_{v \in \Omega_{2}} P\left(\bar{V}_{v, 2}^{k}-V_{v, 2}^{k}<0\right) \\
& \quad+\sum_{k=1}^{N_{D}} \sum_{r \in \Omega_{3}} P\left(\bar{R}_{r, 2}^{k}-R_{r, 2}^{k}<0\right)
\end{aligned}
$$

where

$$
\bar{W}_{w, 2}^{k}=\max _{\substack{(i, j) \neq(w, k) \\ j, h=1, \ldots, N_{D} \\ m=1,2}}\left(W_{i, m}^{j}, W_{w, 1}^{k}, V_{v, m}^{h}, R_{r, m}^{h}\right)
$$

$$
\bar{V}_{v, 2}^{k}=\max _{\substack{(i, j) \neq(v, k) \\ j, h=1, \ldots, N_{D} \\ m=1,2}}\left(W_{w, m}^{h}, V_{i, m}^{j}, V_{v, 1}^{k}, R_{r, m}^{h}\right)
$$

and

$$
\bar{R}_{r, 2}^{k}=\max _{\substack{(i, j) \neq(r, k) \\ j, h=1, \ldots, N_{D} \\ m=1,2}}\left(W_{w, m}^{h}, V_{v, m}^{h}, R_{i, m}^{j}, R_{r, 1}^{k}\right)
$$

The conditional BER $P_{\Omega_{1}, \Omega_{2}, \Omega_{3}}(\varepsilon, \Theta=i), i=1,2,3$, can be computed ${ }^{1}$ as (21), (22), and (23) on the next page, where $\left(G_{1} \cup G_{2}\right)=\Omega$ means that $G_{1}$ and $G_{2}$ are two disjoint subsets of $\Omega$ and the union of those disjoint subsets is $\Omega$ and the set $G$ is defined as $G=\left((x, y): x \in\left(\Omega_{1} \cup \Omega_{2} \cup\{0\}\right), y \in\left\{1,2 \ldots, N_{D}\right\}\right)$. Obviously, the average BER with a given threshold $\theta_{\mathrm{r}}^{\text {th }}$ can be expressed as

$$
\begin{aligned}
& \operatorname{BER}\left(\theta_{\mathrm{r}}^{\text {th }}\right)=\sum_{\Omega_{1} \in \mathcal{P}(\mathcal{S})} \sum_{\Omega_{2} \in \mathcal{P}\left(\mathcal{S} \backslash \Omega_{1}\right)} \sum_{i=1}^{3} \\
& P_{\Omega_{1}, \Omega_{2}, \Omega_{3}}(\varepsilon, \Theta=i) P\left(\Omega_{1}, \Omega_{2}, \Omega_{3}\right) \text { ， }
\end{aligned}
$$

where $\mathcal{P}(\Omega)$ denotes the power set of $\Omega$. The set $\mathcal{S}=$ $\{1, \ldots, K\}$.

\subsection{Optimal and Approximate Thresholds}

Given the closed-form expression of the average BER in (24), one can choose the threshold $\theta_{\mathrm{r}}^{\text {th }}$ to minimize the average BER of the system by using the MATLAB Optimization Toolbox. The optimization problem can be set up as follows:

$$
\widehat{\theta}_{\mathrm{r}}^{\text {th }}=\arg \min _{\theta_{\mathrm{r}}^{\text {th }}} \operatorname{BER}\left(\theta_{\mathrm{r}}^{\text {th }}\right) .
$$

It is clear from (24) that the system need to collect information on the average SNRs of all the transmission links to find the optimal thresholds. Unfortunately, an close-form solution for optimal threshold values is very difficult, if not impossible, to find. Therefore, to further reduce the complexity of the system ${ }^{2}$, in what follows, we propose approximate thresholds and prove that by using those thresholds, the system can achieve the maximum diversity order.

Lemma 1: If the relays use the threshold $\theta_{\mathrm{r}}^{\text {th }}=Q \log c \gamma$ where $\gamma=E_{0} / N_{0}$, the system achieves a full diversity order of $K+N_{D}$ for any $Q \geq N_{D}$ and a positive constant c.

Proof: To simplify our derivation, we consider the i.i.d. case, i.e., $\bar{\gamma}_{0, i}^{1}=\bar{\gamma}_{i, K+1}^{k}=\bar{\gamma}_{0, K+1}^{k}=\bar{\gamma}_{0}, i=$ $1, \ldots, K, k=1, \ldots, N_{D}$ where $\bar{\gamma}_{0}=E_{0} \sigma_{0}^{2} / N_{0}$. Since $\theta_{\mathrm{r}}^{\text {th }}=Q \log c \gamma$ and $\lim _{\bar{\gamma}_{0} \rightarrow \infty} \frac{1-\left(\frac{1}{c \bar{\gamma}}\right)^{\frac{Q}{1+\bar{\gamma}_{0}}}}{\log (\bar{\gamma}) / \bar{\gamma}}=Q \frac{\bar{\gamma}_{0}}{\bar{\gamma}}=Q \sigma_{0}^{2}$,

\footnotetext{
${ }^{1}$ The pdfs of $\bar{W}_{w, 2}^{k}, \bar{V}_{v, 2}^{k}$ and $\bar{R}_{r, 2}^{k}$ are given in Appendix A.

${ }^{2}$ By using the approximate thresholds, besides the information collection, the system does not need to find the optimal thresholds centrally and send to the relays, hence, reducing the complexity and implementation costs of the system.
} 


$$
\begin{aligned}
& P_{\Omega_{1}, \Omega_{2}, \Omega_{3}}(\varepsilon, \Theta=1)=N_{D}\left(\left|\Omega_{1}\right|+1\right) \sum_{l=0}^{N_{D}\left(K+\left|\Omega_{3}\right|+1\right)-1}\left(\begin{array}{c}
N_{D}\left(K+\left|\Omega_{3}\right|+1\right)-1 \\
l
\end{array}\right) \\
& \sum_{\substack{i \in\left(\Omega_{1} \cup \Omega_{2} \cup\{0\}\right) \\
h \in\left\{1, \ldots, N_{D}\right\}}} \sum_{\substack{\left.G_{1} \cup G_{2}\right)=(G \backslash\{(i, h)\})\\
}} \\
& \left.\left((-1)^{N_{D}\left(K+\left|\Omega_{3}\right|+1\right)+\left|G_{2}\right|-l-1} \frac{1}{N_{0}\left(1+\bar{\gamma}_{i, K+1}^{h}\right)}\right)\left(\frac{1}{\sum_{(t, k) \in G_{2}} \frac{1}{N_{0}\left(1+\bar{\gamma}_{t, K+1}^{k}\right)}+\frac{1}{N_{0}\left(1+\bar{\gamma}_{i, K+1}^{h}\right)}+\frac{N_{D}\left(K+\left|\Omega_{3}\right|+1\right)-l}{N_{0}}}\right)\right] \\
& +\frac{N_{D}\left(\left|\Omega_{1}\right|+1\right)\left(N_{D}\left(K+\left|\Omega_{3}\right|+1\right)-1\right)}{N_{0}} \sum_{l=0}^{N_{D}\left(K+\left|\Omega_{3}\right|+1\right)-2}\left(\begin{array}{c}
N_{D}\left(K+\left|\Omega_{3}\right|+1\right)-2 \\
l
\end{array}\right)\left[\sum_{\left(G_{1} \cup G_{2}\right)=G}\right. \\
& \left.\left((-1)^{N_{D}\left(K+\left|\Omega_{3}\right|+1\right)+\left|G_{2}\right|-l-2}\right)\left(\frac{1}{\sum_{(t, k) \in G_{2}} \frac{1}{N_{0}\left(1+\bar{\gamma}_{t, K+1}^{k}\right)}+\frac{N_{D}\left(K+\left|\Omega_{3}\right|+1\right)-l}{N_{0}}}\right)\right]
\end{aligned}
$$

$$
\begin{aligned}
P_{\Omega_{1}, \Omega_{2}, \Omega_{3}}(\varepsilon, \Theta=2)= & \sum_{l=0}^{N_{D}\left(K+\left|\Omega_{3}\right|+1\right)}\left(\begin{array}{c}
N_{D}\left(K+\left|\Omega_{3}\right|+1\right) \\
l
\end{array}\right) \sum_{\substack{v \in \Omega_{2} \\
h \in\left\{1, \ldots, N_{D}\right\}}} \sum_{(i, j) \in G \backslash\{(v, h)\}} \sum_{\left(G_{1} \cup G_{2}\right)=G \backslash\{(v, h),(i, j)\}} \\
& \left(\frac{1}{\left.\left.\sum_{(t, k) \in G_{2}} \frac{1}{N_{0}\left(1+\bar{\gamma}_{t, K+1}^{k}\right)}+\frac{1}{N_{0}\left(1+\bar{\gamma}_{i, K+1}^{j}\right)}+\frac{1}{N_{0}\left(1+\bar{\gamma}_{v, K+1}^{h}\right)}+\frac{N_{D}\left(K+\left|\Omega_{3}\right|+1\right)-l}{N_{0}\left(K+\left|G_{2}\right|+1\right)+\left|\Omega_{3}\right|-l}\right)\right]}\right) \\
& +\frac{1}{N_{0}} \sum_{l=0}^{N_{D}\left(K+\left|\Omega_{3}\right|+1\right)-1}\left(\begin{array}{c}
N_{D}\left(K+\left|\Omega_{3}\right|+1\right)-1 \\
l
\end{array}\right) \sum_{\substack{v \in \Omega_{2} \\
h \in\left\{1, \ldots, N_{D}\right\}}}\left[\sum_{\left(G_{1} \cup G_{2}\right)=G \backslash\{v, h\}}\right. \\
& \left.\left((-1)^{N_{D}\left(K+\left|\Omega_{3}\right|+1\right)+\left|G_{2}\right|-l-1}\right)\left(\frac{1}{\sum_{(t, k) \in G_{2}} \frac{1}{N_{0}\left(1+\bar{\gamma}_{t, K+1}^{k}\right)}+\frac{1}{\left(N_{0}\left(1+\bar{\gamma}_{v, K+1}^{h}\right)\right.}+\frac{N_{D}\left(K+\left|\Omega_{3}\right|+1\right)-l}{N_{0}}}\right)\right]
\end{aligned}
$$

$$
\begin{aligned}
& P_{\Omega_{1}, \Omega_{2}, \Omega_{3}}(\varepsilon, \Theta=3)=N_{D}\left|\Omega_{3}\right| \sum_{l=0}^{N_{D}\left(K+\left|\Omega_{3}\right|+1\right)-1}\left(\begin{array}{c}
N_{D}\left(K+\left|\Omega_{3}\right|+1\right)-1 \\
l
\end{array}\right)\left[\sum_{\substack{i \in\left(\Omega_{1} \cup \Omega_{2} \cup\{0\}\right) \\
h \in\left\{1, \ldots, N_{D}\right\}}} \sum_{\left.\left(G_{1} \cup G_{2}\right)=G \backslash\{(i, h)\}\right)}\right.
\end{aligned}
$$

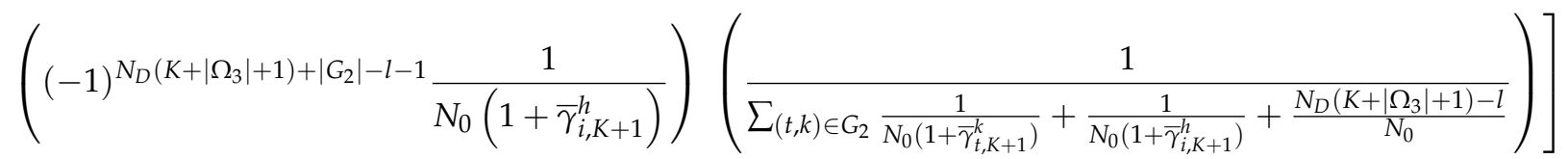

$$
\begin{aligned}
& +\frac{N_{D}\left|\Omega_{3}\right|\left(N_{D}\left(K+\left|\Omega_{3}\right|+1\right)-1\right)}{N_{0}} \sum_{l=0}^{N_{D}\left(K+\left|\Omega_{3}\right|+1\right)-2}\left(\begin{array}{c}
N_{D}\left(K+\left|\Omega_{3}\right|+1\right)-2 \\
l
\end{array}\right)\left[\sum_{\left(G_{1} \cup G_{2}\right)=G}\right. \\
& \left.\left((-1)^{N_{D}\left(K+\left|\Omega_{3}\right|+1\right)+\left|G_{2}\right|-l-2}\right)\left(\frac{1}{\sum_{(t, k) \in G_{2}} \frac{1}{N_{0}\left(1+\bar{\gamma}_{t, K+1}^{k}\right)}+\frac{N_{D}\left(K+\left|\Omega_{3}\right|+1\right)-l}{N_{0}}}\right)\right]
\end{aligned}
$$


it follows from (11) that ${ }^{3}$

$$
\begin{aligned}
P\left(\Omega_{1}, \Omega_{2}, \Omega_{3}\right) \leq & \left(I_{1}\left(\theta_{\mathrm{r}}^{\text {th }}, \bar{\gamma}_{0, i}\right)\right)^{\left|\Omega_{3}\right|}\left(I_{2}\left(\theta_{\mathrm{r}}^{\text {th }}, \bar{\gamma}_{0, i}\right)\right)^{\left|\Omega_{2}\right|} \\
& \triangleq(\log (\bar{\gamma}) / \bar{\gamma})^{\left|\Omega_{3}\right|}\left(1 / \bar{\gamma}^{Q+1}\right)^{\left|\Omega_{2}\right|} .
\end{aligned}
$$

On the other hand, the conditional BER $P_{\Omega_{1}, \Omega_{2}, \Omega_{3}}(\varepsilon)=$ $\sum_{i=1}^{3} P_{\Omega_{1}, \Omega_{2}, \Omega_{3}}(\varepsilon, \Theta=i)$ can be evaluated from the large SNR behavior by considering the value of the first nonzero order derivative of the PDF at the origin [11]. According to [12], one can verify that

$$
\begin{aligned}
& P_{\Omega_{1}, \Omega_{2}, \Omega_{3}}(\varepsilon, \Theta=1) \triangleq\left(1 / \bar{\gamma}_{0}\right)^{N_{D}\left(\left|\Omega_{1}\right|+\left|\Omega_{2}\right|+1\right)}, \\
& P_{\Omega_{1}, \Omega_{2}, \Omega_{3}}(\varepsilon, \Theta=2)
\end{aligned}
$$

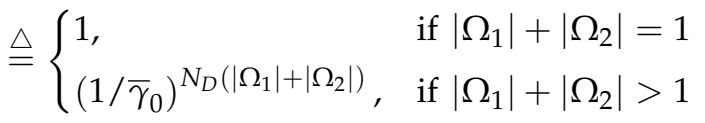

$$
\begin{aligned}
& P_{\Omega_{1}, \Omega_{2}, \Omega_{3}}(\varepsilon, \Theta=3) \triangleq\left(1 / \bar{\gamma}_{0}\right)^{N_{D}\left(\left|\Omega_{1}\right|+\left|\Omega_{2}\right|+1\right)} .
\end{aligned}
$$

Thus, one has

$$
\begin{aligned}
& P_{\Omega_{1}, \Omega_{2}, \Omega_{3}}(\varepsilon) \\
& \triangleq\left\{\begin{array}{l}
\left(1 / \bar{\gamma}_{0}\right)^{K+N_{D}}, \text { if }\left|\Omega_{2}\right|=0 \\
\left(1 / \bar{\gamma}_{0}\right)^{K+Q}, \text { if }\left|\Omega_{1}\right|=0 \text { and }\left|\Omega_{2}\right|=1 \\
\left(1 / \bar{\gamma}_{0}\right)^{\left|\Omega_{3}\right|+\left|\Omega_{2}\right|(Q+1)+N_{D}\left(\left|\Omega_{1}\right|+\left|\Omega_{2}\right|\right)}, \\
\text { if }\left|\Omega_{1}\right|>0 \text { and }\left|\Omega_{2}\right|>0
\end{array}\right.
\end{aligned}
$$

Therefore, for sufficiently large values of SNR and $\theta_{\mathrm{r}}^{\text {th }}=Q \log c \gamma$ where $Q \geq N_{D}$, it follows from (24) that $\operatorname{BER}\left(\theta_{\mathrm{r}}^{\text {th }}\right) \triangleq\left(1 / \bar{\gamma}_{0}\right)^{K+N_{D}}$. So Lemma 1 is proved.

\section{Simulation Results}

This section presents analytical and simulation results for the BER performance of different noncoherent DF cooperative systems. In all simulations, the noise components at the destination and relays are modeled as i.i.d. zero-mean complex Gaussian random variables with variances 1 . The source and relays have an equal transmit power, i.e., $E_{i}=E, i=0, \ldots, K$. The channels between any two antennas are assumed to be Rayleigh flat fading. It is also assumed that the variances of Rayleigh fading channels between any two antennas of two nodes are identical, i.e., $\left(\sigma_{i, j}^{k}\right)^{2}=\sigma_{i, j}^{2}$.

Figure 2 plots the average BERs of the proposed scheme, PL scheme and the scheme in [6] in a tworelay system with single-antenna equipped destination when the variances of Rayleigh fading channels are set to be $2 \sigma_{0, i}^{2}=0.1 \sigma_{i, K+1}^{2}=5 \sigma_{0, K+1}^{2}=1, i=1,2$. It is note that the system with single-antenna equipped destination is a special case of the one with multipleantenna equipped destination. From the figure, both

\footnotetext{
${ }^{3}$ With two positive real functions $f(x)$ and $g(x)$, we say $f(x) \triangleq$ if $\limsup _{x \rightarrow \infty} \frac{f(x)}{g(x)}=d$ where $d<\infty$ is a positive constant.
}

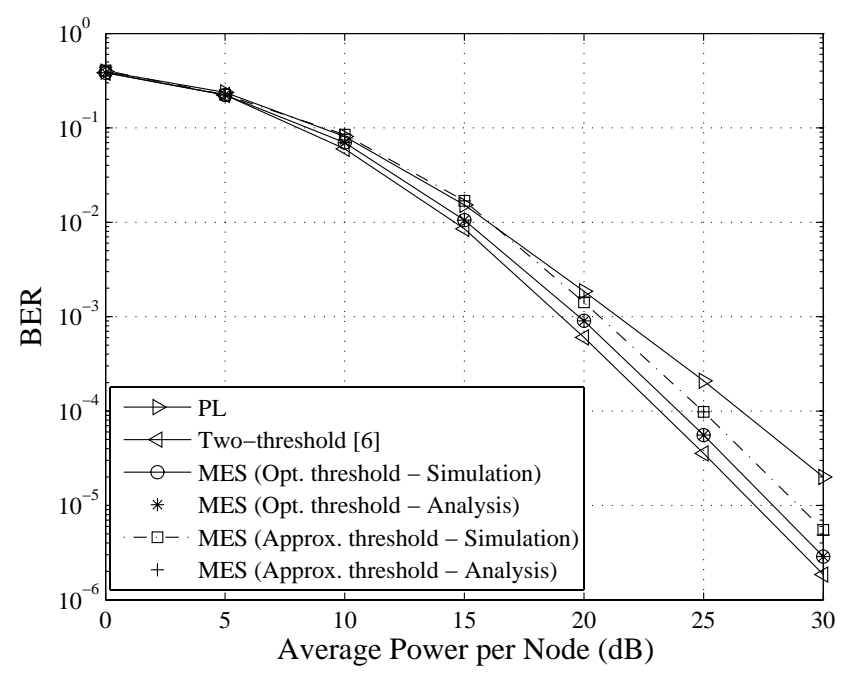

Figure 2. BERs of a two-relay network with different schemes when $2 \sigma_{0, i}^{2}=0.1 \sigma_{i, K+1}^{2}=5 \sigma_{0, K+1}^{2}=1$.

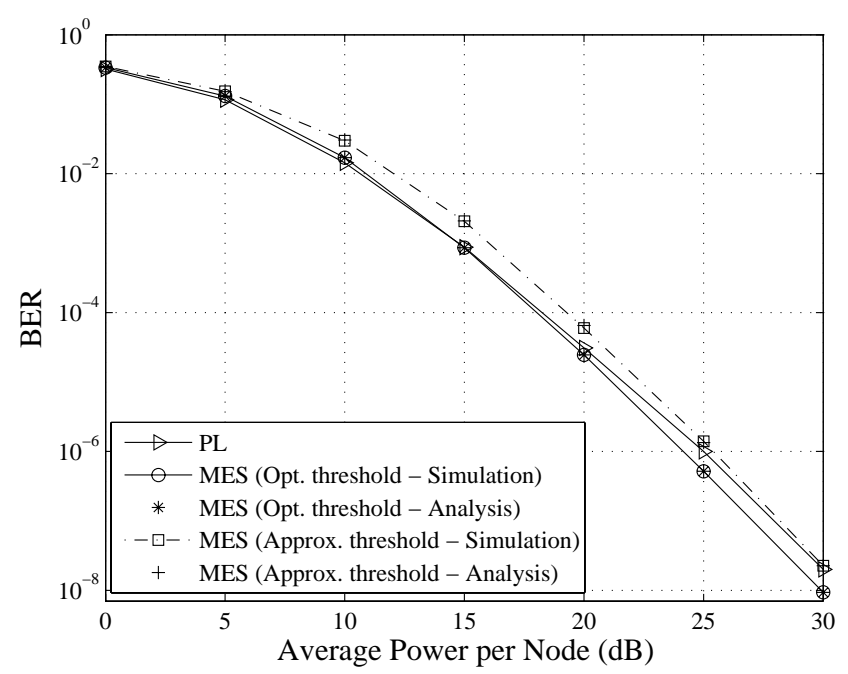

Figure 3. BERs of a three-relay network with different schemes when $\sigma_{0, i}^{2}=\sigma_{i, K+1}^{2}=\sigma_{0, K+1}^{2}=1$.

the analytical (shown as marker symbols) and simulation (shown in line with marker symbols) results are identical, hence verifying our analysis in Section 3. The figure also shows that the BER of the proposed scheme is significantly better than the BER of the PL scheme. It is institutively clear since the PL scheme suffers from the error propagation. The scheme in [6] outperforms the other two schemes due to the fact that the destination in [6] combines all the signals from the retransmitting relays besides dealing with the problem of error propagation. However, the proposed scheme does not require any statistical information of the fading channels to perform a detection. Such the information is required for the PL scheme and the scheme in [6].

Figure 3 presents the average BERs obtained by simulation and analysis for two different schemes in a three-relay cooperative system. Here $\sigma_{0, i}^{2}=\sigma_{i, K+1}^{2}=$ $\sigma_{0, K+1}^{2}=1, i=1,2,3$. The figure again confirms the 


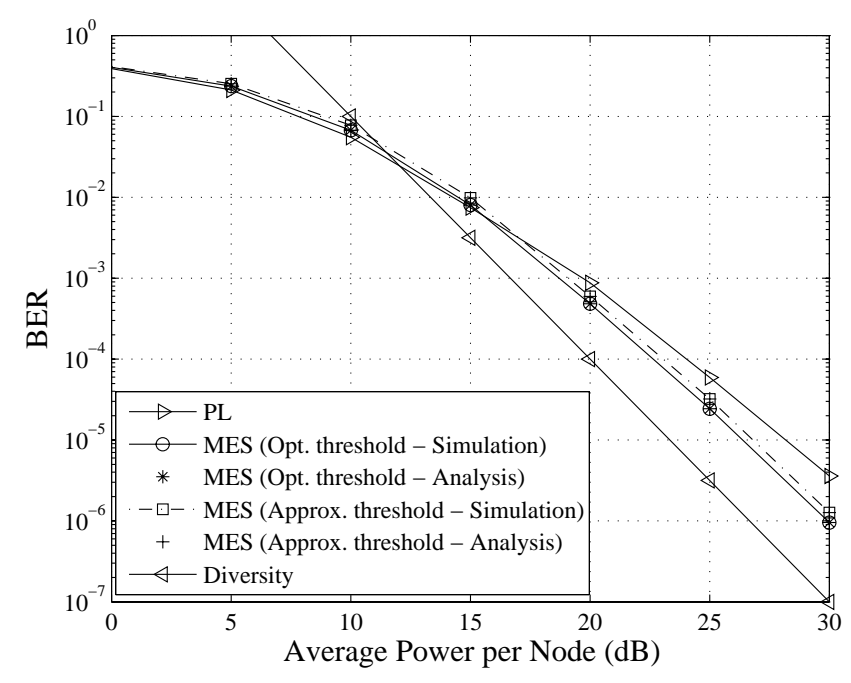

Figure 4. BERs of a single relay network with different schemes when the destination is equipped with two antennas and $0.2 \sigma_{0, i}^{2}=2 \sigma_{i, K+1}^{2}=10 \sigma_{0, K+1}^{2}=1$.

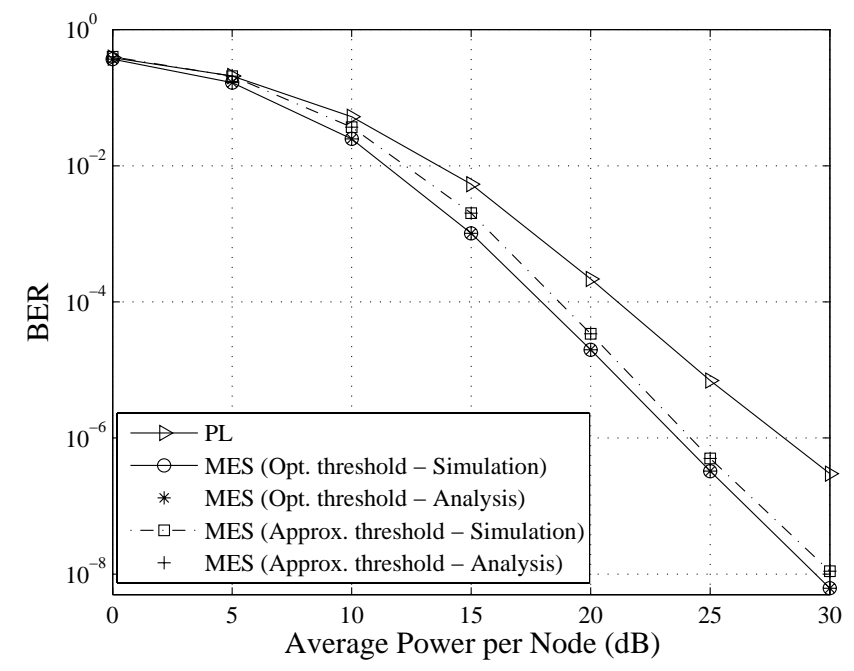

Figure 5. BERs of a two-relay network with different schemes when the destination is equipped with two antennas and $0.2 \sigma_{0, i}^{2}=2 \sigma_{i, K+1}^{2}=10 \sigma_{0, K+1}^{2}=1$.

analysis performed in Section 3. At sufficient large values of SNR, the proposed scheme yields a superior performance compared to the PL scheme.

Figures 4 and 5 plot the average BER of the proposed scheme for a single-relay network and a tworelay network, respectively, in which the destination is equipped with two antennas. Here $0.2 \sigma_{0,1}^{2}=2 \sigma_{1,2}^{2}=$ $10 \sigma_{0,2}^{2}=1$ for a single-relay network and $0.2 \sigma_{0,1}^{2}=$ $0.2 \sigma_{0,2}^{2}=2 \sigma_{1,3}^{2}=2 \sigma_{2,3}^{2}=10 \sigma_{0,3}^{2}=1$ for a two-relay network. The figures show that the exact analysis and simulation results are the same. One can again observe that the proposed scheme outperforms the PL scheme. By using the approximate thresholds, the system can achieve a maximum diversity order of 3 for a singlerelay network with two-antenna equipped destination as confirmed in Figure 4.

\section{Conclusion}

This paper studies the maximum energy selection receiver for an adaptive decode-and-forward (DF) relaying system with BFSK signals. The considered relaying system consists of a source, a relay, and destination in which the source and relays are equipped with a single antenna and the destination is equipped with multiples antennas. A closed-form BER expression is obtained and used to choose the optimal thresholds to minimize the average BER. Approximate thresholds are proposed and the diversity order is verified. Performance comparison reveals that the proposed scheme outperforms the PL scheme.

\section{ACKNOWLEDGEMENT}

This research is funded by Vietnam National Foundation for Science and Technology Development (NAFOSTED) under grant number 102.04-2012.33.

\section{Appendix A \\ PDFS OF $\bar{W}_{w, 2}, \bar{V}_{v, 2}$, AND $\bar{R}_{r, 2}$ RANDOM VARIABLES}

The pdf of $\bar{W}_{w, 2}, \bar{V}_{v, 2}$, and $\bar{R}_{r, 2}$ can be found, respectively, as follows:

$$
\begin{aligned}
& f_{\bar{W}_{w, 2}^{k}}(x)=\frac{\mathrm{d}}{\mathrm{d} x} P\left(\bar{W}_{w, 2}^{k}<x\right)=\sum_{\substack{i \in\left(\Omega_{1} \cup \Omega_{2} \cup\{0\}\right) \\
h=1, \ldots, N_{D}}} f_{i, 1}^{h}(x) \\
& \times \prod_{\substack{j \in\left(\Omega_{1} \cup \Omega_{2} \cup\{0\}\right) \\
l=1, \ldots, N_{D} \\
(j, l) \neq(i, h)}} F_{j, 1}^{l}(x)\left(F_{1,2}^{1}(x)\right)^{N_{D}\left(K+\left|\Omega_{3}\right|+1\right)-1} \\
& +\frac{N_{D}\left(K+\left|\Omega_{3}\right|+1\right)-1}{N_{0}} \mathrm{e}^{-x / N_{0}}\left(F_{1,2}^{1}(x)\right)^{N_{D}\left(K+\left|\Omega_{3}\right|+1\right)-2} \\
& \times \prod_{\substack{j \in\left(\Omega_{1} \cup \Omega_{2} \cup\{0\}\right) \\
h=1, \ldots, N_{D}}} F_{j, 1}^{h}(x) \\
& f_{\bar{V}_{v, 2}^{k}}(x)=\frac{\mathrm{d}}{\mathrm{d} x} P\left(\bar{V}_{v, 2}^{k}<x\right)=\sum_{\substack{i \in\left(\Omega_{1} \cup \Omega_{2} \cup\{0\}\right) \\
h=1, \ldots, N_{D} \\
(i, h) \neq(v, k)}} f_{i, 1}^{h}(x) \\
& \times \prod_{\substack{j \in\left(\Omega_{1} \cup \Omega_{2} \cup\{0\}\right) \\
l=1, \ldots, N_{D}}} F_{j, 1}^{l}(x)\left(F_{1,2}^{1}(x)\right)^{N_{D}\left(K+\left|\Omega_{3}\right|+1\right)} \\
& (j, l) \neq\{(v, k),(i, h)\} \\
& +\frac{N_{D}\left(K+\left|\Omega_{3}\right|+1\right)}{N_{0}} \mathrm{e}^{-x / N_{0}}\left(F_{1,2}^{1}(x)\right)^{N_{D}\left(K+\left|\Omega_{3}\right|+1\right)-1} \\
& \times \prod_{\substack{j \in\left(\Omega_{1} \cup \Omega_{2} \cup\{0\}\right) \\
h=1, \ldots, N_{D}}} F_{j, 1}^{h}(x) \\
& \begin{array}{l}
h=1, \ldots, N_{D} \\
(j, h) \neq(v, k)
\end{array}
\end{aligned}
$$




$$
\begin{gathered}
f_{\bar{R}_{r, 2}^{k}}(x)=\frac{\mathrm{d}}{\mathrm{d} x} P\left(\bar{R}_{r, 2}^{k}<x\right)=\sum_{\substack{i \in\left(\Omega_{1} \cup \Omega_{2} \cup\{0\}\right) \\
h=1, \ldots, N_{D}}} f_{i, 1}^{h}(x) \\
\times \prod_{\substack{j \in\left(\Omega_{1} \cup \Omega_{2} \cup\{0\}\right) \\
l=1, \ldots, N_{D} \\
(j, l) \neq(i, h)}} F_{j, 1}^{l}(x)\left(F_{1,2}^{1}(x)\right)^{N_{D}\left(K+\left|\Omega_{3}\right|+1\right)-1} \\
+\frac{N_{D}\left(K+\left|\Omega_{3}\right|+1\right)-1}{N_{0}} \mathrm{e}^{-x / N_{0}}\left(F_{1,2}^{1}(x)\right)^{N_{D}\left(K+\left|\Omega_{3}\right|+1\right)-2} \\
\times \prod_{\substack{j \in\left(\Omega_{1} \cup \Omega_{2} \cup\{0\}\right) \\
h=1, \ldots, N_{D}}} F_{j, 1}^{h}(x)
\end{gathered}
$$

where $F_{k, 1}^{l}(x)=1-\mathrm{e}^{-x /\left(N_{0}\left(1+\bar{\gamma}_{k, K+1}^{l}\right)\right)}$ and $F_{k, 2}^{l}(x)=1-$ $\mathrm{e}^{-x / N_{0}}$.

\section{REFERENCES}

[1] M. K. Simon and M.-S. Alouini, Digital Communication over Fading Channels. Wiley, 2005.

[2] R. Annavajjala, P. Cosman, and L. Milstein, "On the performance of optimum noncoherent amplify-and-forward reception for cooperative diversity," in IEEE Military Communications Conference, MILCOM 2005, vol. 5, October 2005 , pp. $3280-3288$.

[3] D. Chen and J. Laneman, "Modulation and demodulation for cooperative diversity in wireless systems," IEEE Transactions on Wireless Communications, vol. 5, pp. 17851794, July 2006.

[4] M. Souryal, "Non-coherent amplify-and-forward generalized likelihood ratio test receiver," IEEE Transactions on Wireless Communications, vol. 9, no. 7, pp. 2320-2327, 2010.

[5] G. Farhadi and N. Beaulieu, "A low complexity receiver for noncoherent amplify-and-forward cooperative systems," IEEE Transactions on Communications, vol. 58, pp. 2499-2504, August 2010.

[6] H. Nguyen and H. Nguyen, "Adaptive relaying in noncoherent cooperative networks," IEEE Transactions on Signal Processing, vol. 58, pp. 3938-3945, March 2010.

[7] H. X. Nguyen and H. H. Nguyen, "Selection combining for noncoherent decode-and-forward relay networks," EURASIP Journal on Wireless Communications and Networking, vol. 2011:106, 2011.

[8] J. Laneman and G. W. Wornell, "Distributed space-timecoded protocols for exploiting cooperative diversity in wireless networks," IEEE Transactions on Information Theory, vol. 49, no. 10, pp. 2415-2425, 2003.

[9] J. Laneman, D. Tse, and G. W., "Cooperative diversity in wireless networks: Efficient protocols and outage behavior," IEEE Transactions on Information Theory, vol. 50, no. 12, pp. 3062-3080, 2004

[10] H. Nguyen, C. Ho, C. D. T. Thai, and D. Nguyen, "Noncoherent decode-and-forward cooperative systems with maximum energy selection," in International Conference on Advanced Technologies for Communications (ATC 2013), October 2013, pp. 136-140.

[11] Z. Wang and G. Giannakis, "A simple and general parameterization quantifying performance in fading channels," IEEE Transactions on Communications, vol. 51, pp. $1389-1398,2003$.

[12] Y. Zhao, R. Adve, and T. J. Lim, "Symbol error rate of selection amplify-and-forward relay systems," IEEE Communications Letters, vol. 10, pp. 757-759, 2006.

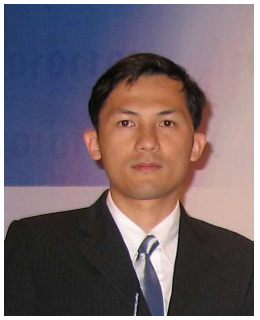

Ha X. Nguyen received the B.Eng. degree from Posts and Telecommunications Institute of Technology, Hochiminh City, Vietnam, in 2003, the M.Sc. degree from Korea Advanced Institute of Science and Technology, Daejeon, Korea, in 2007, and the Ph.D. degree from the University of Saskatchewan, Saskatoon, SK, Canada, in 2011. Since August 2011, he has joined Tan Tao University as an Assistant Professor in the School of Engineering. His research interests span the areas of unequal error protection in digital communications and cooperative wireless networks.

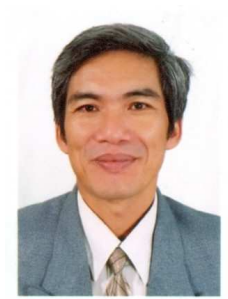

Ho Van Cuu was born in 1964 in Quang Ngai City, Vietnam. He graduated the Bachelor Engineer Degree in telecommunications from Ho Chi Minh City University of Technology, and Master degree in electronic telecommunications from Ha Noi University of Technology in 1987 and 1997, respectively. He received $\mathrm{Ph} . \mathrm{D}$ degree in telecommunications from Post and Telecommunications Institute Technology PTIT in 2007. Currently, he is Head of department of Electronics and Telecommunications of Sai Gon University (SGU). His research interests include mobile communications, digital multiplexer and Radio communication networks.

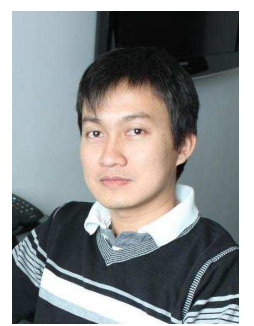

Chan Dai Truyen Thai received his B.S. from Posts and Telecommunications Institute of Technology, Vietnam, in 2003, his MSc. from Korea Advanced Institute of Science and Technology (KAIST), South Korea, in 2008 and Ph.D. from Aalborg University, Denmark, in 2012. He is now a post-doctoral researcher in Singapore University of Technology and Design (SUTD). His interest topics include: cooperative/relaying communications, femtocell, communication for high-speed vehicles, 